\title{
Adesão a um programa de aconselhamento nutricional para adultos com excesso de peso e comorbidades
}

\author{
Adherence to a nutritional counseling \\ program for adults with excess \\ weight and comorbidities
}

\author{
Norma Gonzaga GUIMARÃES 1 \\ Eliane Said DUTRA ${ }^{2}$ \\ Marina Kiyomi ITO2 \\ Kenia Mara Baiocchi de CARVALHO²
}

\section{RE S U M O}

\section{Objetivo}

Investigar adesão a programa de aconselhamento nutricional em grupo para indivíduos com excesso de peso e comorbidades.

\section{Métodos}

Estudo analítico de intervenção controlada e aberta. Oitenta adultos, de ambos os sexos, com índice de massa corporal entre 25 e $35 \mathrm{~kg} / \mathrm{m}^{2}$, portadores de dois ou mais fatores de risco cardiovascular associados foram alocados aleatoriamente em dois grupos para acompanhamento por três meses. O grupo de intervenção com aconselhamento nutricional em grupo recebeu atendimento individual e participou de seis reuniões grupais para discussão sobre alimentação saudável e atividade física, com dinâmicas e método participativo. O grupo-controle, com atendimento padrão individual, foi assistido em três consultas ambulatoriais. Consideraram-se repercussões dietéticas antes e após a intervenção e assiduidade às reuniões como parâmetros de adesão ao tratamento.

\section{Resultados}

Trinta e três participantes concluíram o estudo. Do total inicial, 45,8\% e 40,7\% do grupo de intervenção com aconselhamento nutricional em grupo e do grupo-controle com atendimento padrão individual, respectivamente, atingiram nível ótimo de assiduidade. Os fatores mais citados como barreiras à adesão ao tratamento foram fazer refeições fora de casa (46,7\% - grupo-controle com atendimento padrão individual) e dificuldade

1 Secretaria de Estado de Saúde do Distrito Federal, Programa de Residência em Nutrição Clínica. Brasília, DF, Brasil.

2 Universidade de Brasília, Faculdade de Ciências da Saúde, Departamento de Nutrição. Campus Universitário Darcy Ribeiro, 70910-900, Brasília, DF, Brasil. Correspondência para/Correspondence to: K.M.B. CARVALHO. E-mail: <kenia@unb.br>. 
324 | N.G. GUIMARÃES et al.

em aplicar os conhecimentos na prática, principalmente em eventos sociais (33,3\% - grupo de intervenção com aconselhamento nutricional em grupo). Verificou-se aumento significativo do percentual médio do uso de temperos naturais e do número de refeições ao dia nos dois grupos após a intervenção. Porém, não houve diminuição significativa do consumo energético intra ou entre os grupos.

\section{Conclusão}

Apesar da abrangência do conteúdo e do emprego de um método participativo no grupo de intervenção, a adesão foi insuficiente para alterar significativamente os principais parâmetros dietéticos estudados. O aprofundamento da abordagem comportamental, continuada e multiprofissional deve ser objeto de mais investigações.

Termos de indexação: Aconselhamento nutricional. Adesão. Adultos. Obesidade.

\section{A B S T R A C T}

\section{Objective}

The objective of this study was to verify adherence to a nutritional group counseling program for patients with excess weight and comorbidities.

\section{Methods}

This was an analytical, open, controlled, intervention study. Eighty adults from both genders with a body mass index ranging from 25 to $35 \mathrm{~kg} / \mathrm{m}^{2}$ with two or more associated cardiovascular risk factors were randomly allocated to two groups for a three-month follow-up. The intervention group with nutritional group counseling received personalized care and participated in six group meetings to discuss healthy eating and physical activity, with group dynamics and participatory method. The control group was given standard personalized care in three outpatient visits. The parameters of adherence to treatment were the dietary repercussions before and after the intervention and attendance to the meetings.

\section{Results}

Thirty-three participants concluded the study. In all, 45.8\% and 40.7\% of intervention group and control group, respectively, had excellent attendance. The main barriers to adherence to treatment were the need of eating out (46.7\% - control group) and the difficulty of putting their knowledge to use, especially in social occasions (33.3\% - intervention group). There was a significant increase in the mean percentage use of natural seasonings and in the number of daily meals of both groups after the intervention. However, there was no significant decrease in energy intake within groups or between groups.

\section{Conclusion}

Despite the breadth of the content and the use of a participatory method in the intervention group, adherence was not enough to change the main dietary parameters significantly. A deeper, continuous and multidisciplinary behavioral approach should be object of further investigations.

Indexing terms: Nutritional counseling. Adherence. Adults. Obesity.

\section{N T R O D U ÇÃ O}

A obesidade é uma doença crônica relacionada às mudanças de comportamento, principalmente com relação à alimentação e à atividade física. Afeta desfavoravelmente a saúde e a qualidade de vida do indivíduo e, consequentemente, gera mais custos para a sociedade e para o governo ${ }^{1}$.

No Brasil, ao longo das últimas décadas, o processo de transição nutricional foi acompanhado por aumento significativo da obesidade e diminuição das taxas de desnutrição da população pobre e rica do País. Inicialmente, o problema atingia, em particular, as mulheres; dados mais recentes, contudo, mostram um aumento da obesidade - em torno de $40 \%$ - de forma equilibrada entre os gêneros na população adulta².

O tratamento da obesidade, baseado em dieta e atividade física, com o objetivo de uma perda ponderal de $5 \%$ a $10 \%$ do peso inicial, parece ser medida efetiva para controlar as alte- 
rações metabólicas e os fatores de risco cardiovascular $^{3}$. Porém, observam-se altas taxas de insucesso desse tratamento principalmente pela baixa adesão aos programas dietéticos, dificuldade de emagrecimento e precária manutenção dos resultados ${ }^{4}$. Inelmen et al. ${ }^{5}$ apontam que, entre os fatores que podem dificultar a adesão ao tratamento da obesidade, destacam-se os problemas pessoais, a ausência de apoio familiar, a falta de motivação e os resultados negativos na perda de peso durante o tratamento. Segundo os mesmos autores, estudos clínicos têm demonstrado taxa de desistência de até $80 \%$.

Acredita-se que novas estratégias de atendimento nutricional devam ser desenvolvidas para promover uma motivação autônoma. O paciente torna-se, assim, capaz de identificar barreiras para o autocontrole e desenvolver mecanismos para superá-las. O indivíduo deverá prevenir recaídas, fazer melhores escolhas diante dos desafios cotidianos (empowerment) e adquirir a habilidade para resolver problemas e, portanto, obter melhores resultados no tratamento da obesidade ${ }^{6}$.

O objetivo deste estudo foi avaliar a adesão de adultos portadores de excesso de peso e outros fatores de risco cardiovascular a um programa de aconselhamento nutricional em grupo, por meio da assiduidade às reuniões e repercussões nos parâmetros dietéticos.

\section{MÉ T O D OS}

Trata-se de estudo analítico de intervenção controlada e aberta para o qual foram cadastrados 250 indivíduos no ano de 2005, por meio de anúncio em jornal, rádio e televisão. Desses, 80 foram considerados elegíveis para participar do estudo segundo o critério adotado: ambos os gêneros, idade entre 18 e 65 anos, Índice de Massa Corporal (IMC) entre 25 e $35 \mathrm{~kg} / \mathrm{m}^{2}$ e portadores de dois ou mais fatores de risco cardiovascular associados, como hipertensão arterial, dislipidemia, intolerância à glicose ou diabetes Mellitus. Foram excluídos indivíduos que estivessem participando de outro programa de educação nutricional, que tivessem realizado cirurgia de obesidade ou que fossem portadores de doença que necessite de plano alimentar específico.

Os indivíduos foram aleatoriamente alocados em dois grupos de acompanhamento: Aconselhamento Nutricional em Grupo (ANG), no qual os voluntários receberam acompanhamento individual mensal e participaram de um programa de aconselhamento nutricional em grupo durante três meses $(n=40)$, e Atendimento Padrão Individual (API), representado pelo acompanhamento individual mensal no Ambulatório de Nutrição e Dietética do Hospital Universitário de Brasília $(n=40)$, com consultas de uma hora de duração pelo mesmo período.

Para caracterização da amostra e avaliação dos resultados, foram coletados dados sociodemográficos, antropométricos (peso, estatura e circunferência da cintura) e informações clínicas, como a presença de comorbidades e a medida de pressão arterial de consultório. Para a circunferência da cintura utilizou-se o ponto médio entre a última costela e a espinha ilíaca ântero-posterior e para as demais medidas seguiram-se os procedimentos padrões ${ }^{7,8}$. Os voluntários foram classificados em sedentários ou ativos, sendo considerados ativos os que praticavam alguma atividade física ou exercício programado como caminhada, natação ou ginástica no mínimo três vezes por semana por trinta minutos.

A renda dos participantes foi classificada de acordo com os critérios da Sociedade de Pesquisa de Mercado ${ }^{9}$ em sete classes socioeconômicas: $A 1, A 2, B 1, B 2, C, D$ e $E$, em um gradiente decrescente de poder de compra.

Para avaliação do consumo alimentar, solicitou-se, no início da pesquisa, que os participantes realizassem um registro alimentar de três dias, sendo um deles de final de semana. Repetiu-se o mesmo procedimento ao final do período da intervenção. Os cálculos dos registros alimentares foram realizados com base na Tabela de Composição de Alimentos $^{10}$ e na Tabela para Avaliação de Consumo Alimentar em Medidas Caseiras $^{11}$. 
Verificaram-se, também, características qualitativas do padrão alimentar antes e depois da intervenção por meio de um Questionário de Frequência Alimentar (QFA) simplificado, aplicado pelo pesquisador ou entrevistador treinado para investigar o consumo de frutas, hortaliças e o uso de temperos.

Conceitos relativos ao papel da alimentação na saúde das pessoas foram avaliados por meio de questionário de conhecimento específico aplicado antes e após o período de intervenção, consistindo de 10 questões objetivas, resultando em escore de 0 a 10.

Para participarem do programa de aconseIhamento nutricional em grupo, os voluntários foram divididos em turmas de 20 participantes. Esse programa foi composto por seis reuniões quinzenais com três horas de duração cada uma, totalizando, ao final de três meses, uma carga horária de 18 horas.

Nesses encontros foram abordados os seguintes conteúdos: grupos de alimentos e pirâmide dos alimentos, princípios de uma alimentação saudável, rotulagem nutricional, composição de um prato saudável, dietas da moda e apresentação de documentário sobre a cultura do fast food. Foi empregado método participativo com dinâmicas de grupo ${ }^{12}$, quando os participantes apresentavam seus conhecimentos prévios por meio de jogos, simulação de compra em supermercado ou trabalhos em grupo e os instrutores realizavam uma explanação e promoviam uma análise crítica sobre o tema do dia. Foi fornecido material educativo acerca dos conteúdos apresentados e estratégias de como adequar o comportamento em diferentes situações do cotidiano. Todo o material foi elaborado especificamente para a pesquisa.

Para avaliação da adesão ao tratamento, consideraram-se o registro da assiduidade às consultas e às reuniões em grupo e a avaliação dos parâmetros dietéticos, pesquisados no QFA simplificado, antes e ao final do período da pesquisa. Não se considerou perda ponderal ou outra repercussão clínica na análise da adesão uma vez que o comportamento alimentar precede tais indicadores e foi o foco principal dessa intervenção.

Ao final do estudo, foi solicitado a todos os participantes que respondessem a um questionário sobre fatores que favorecem ou prejudicam o seguimento das orientações nutricionais recebidas durante o tratamento.

Para a análise descritiva e comparação dos grupos no momento inicial do protocolo, utilizaram-se o test $t$ de Student e o qui-quadrado por meio do programa estatístico SAS versão $8.2^{13}$. Os possíveis fatores preditores associados à adesão entre os voluntários que completaram ou não o estudo e as dificuldades relatadas pelos pacientes para um seguimento adequado das recomendações alimentares recebidas durante o tratamento foram verificados por ajustamento de uma regressão logística multivariada. Do resultado do modelo calculou-se a razão de chances. Para avaliar o efeito da intervenção sobre o consumo energético entre os grupos, foram utilizados o teste de análise de variância a dois fatores e o test $t$ pareado para avaliar o efeito intragrupo. Para todas as análises, utilizou-se um nível de significância de 5\%.

O estudo foi aprovado pelo Comitê de Ética em Pesquisa da Faculdade de Ciências da Saúde da Universidade de Brasília, protocolo n 021/2004, e a participação, condicionada à assinatura de termo de consentimento livre e esclarecido.

\section{RESULTADOS}

Após três meses de acompanhamento, dos 80 indivíduos considerados elegíveis, 33 concluíram o estudo (41,2\%). A taxa de desistência foi superior a $50 \%$ entre os participantes de ambos os grupos. Não houve diferença significativa em relação às características sociodemográficas e clínicas dos participantes que saíram e dos que permaneceram no estudo (Figura 1).

Os grupos eram homogêneos, apesar da diferença em relação à idade. O perfil amostral era composto basicamente de mulheres de aproximadamente 50 anos, com elevado grau de instrução e renda, obesidade central e, em sua maioria, 


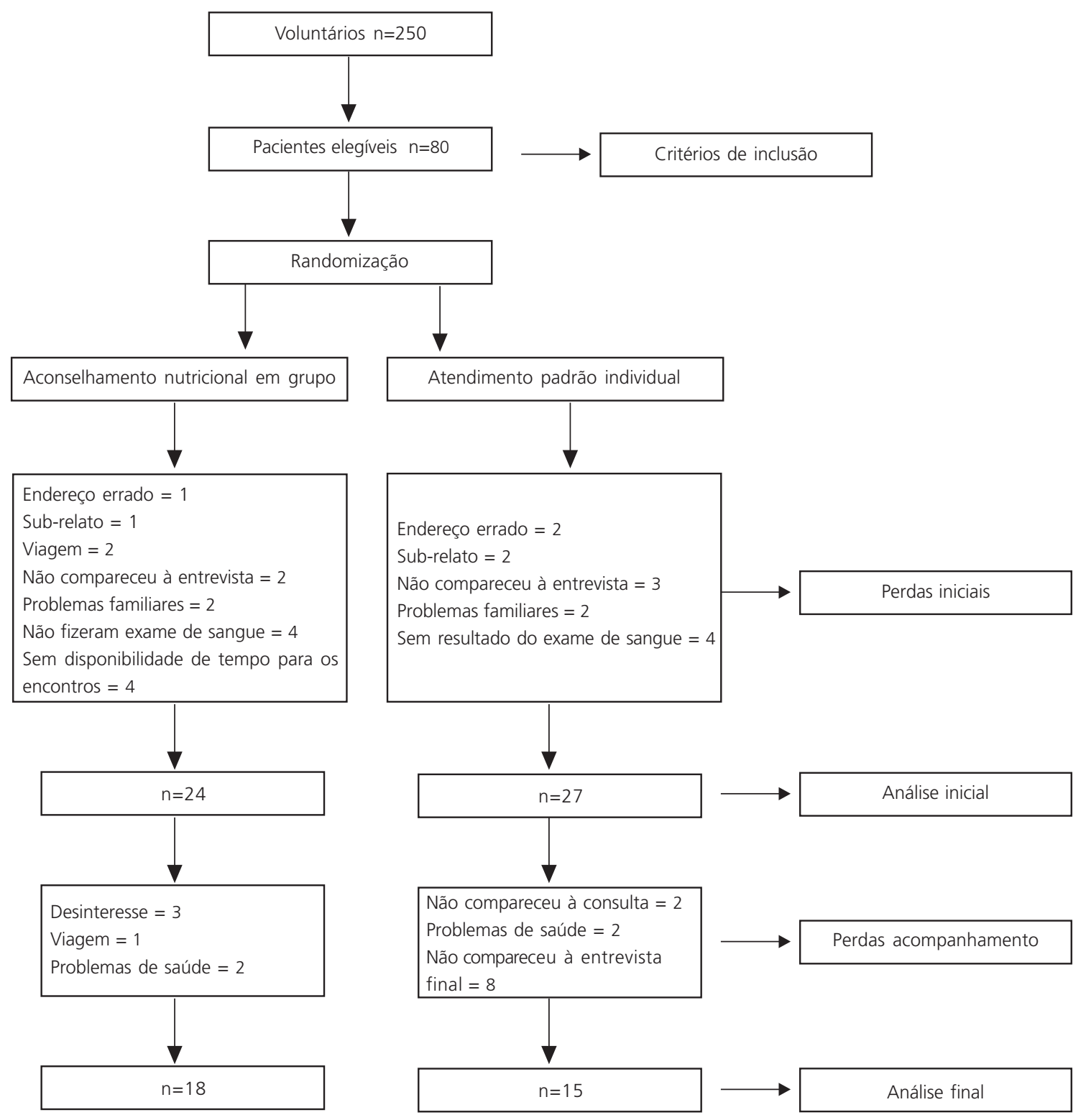

Figura 1. Composição e perdas amostrais dos grupos de estudo. Brasília (DF), 2005.

apresentando hipertensão e dislipidemias (Tabela 1).

Em relação à frequência dos pacientes às reuniões do Atendimento Nutricional em Grupo, houve decréscimo de assiduidade com a progressão do curso, com um ligeiro aumento correspondente ao $4^{\circ}$ encontro, quando se trabalhou com as famílias dos voluntários (Tabela 2).

A análise da assiduidade dos pacientes às consultas individuais revelou que aproxima- damente $40 \%$ dos indivíduos completaram todo o protocolo - três consultas - sem que houvesse diferença entre os grupos (Figura 3).

Os participantes de ambos os grupos apresentaram alto nível de conhecimento das questões avaliadas antes mesmo do início da intervenção, com escore superior a 8,0. Não houve diferença significativa dos resultados quando comparados ao período final da pesquisa (dados não apresentados). 
328 | N.G. GUIMARÃES et al.

Tabela 1. Perfil sociodemográfico, diagnóstico clínico, antropometria, pressão arterial e consumo energético dos grupos de Aconselhamento Nutricional em Grupo (ANG) e de Atendimento Padrão Individual (API) no início do estudo. Brasília (DF), 2005.

\begin{tabular}{|c|c|c|c|}
\hline Variável & ANG $(n=24)$ & API $(n=27)$ & $p$ \\
\hline Gênero & & & 0,562 \\
\hline Feminino (\%) & 66,7 & 74,1 & \\
\hline Idade (anos) & $M=47,8, D P=10,6$ & $M=54,2, D P=7,6$ & 0,018 \\
\hline Grau de escolaridade (\%) & & & 0,358 \\
\hline Ensino Fundamental & 12,5 & 3,7 & \\
\hline Ensino Médio & 33,3 & 37,0 & \\
\hline Ensino Superior (completo) & 54,2 & 59,3 & \\
\hline Prática de atividade fisica referida (\%) & 50,0 & 51,9 & 0,895 \\
\hline Renda $(\%)^{\mathbf{a}}$ & & & 0,375 \\
\hline A1 & 16,7 & 11,1 & \\
\hline A2 & 29,2 & 40,7 & \\
\hline B1 & 25,0 & 11,1 & \\
\hline B2 & 12,5 & 29,6 & \\
\hline C & 12,5 & 7,4 & \\
\hline D & 4,2 & 0,0 & \\
\hline Diagnóstico clínico referido (\%) & & & 0,517 \\
\hline $\mathrm{HAS}^{\mathbf{b}}+\mathrm{DM}^{\mathrm{c}}$ & 8,3 & 18,5 & \\
\hline HAS + Dislipidemias & 62,5 & 48,1 & \\
\hline DM + Dislipidemias & 12,5 & 7,4 & \\
\hline HAS + DM+ Dislipidemias & 16,7 & 25,9 & \\
\hline Peso corporal $(\mathrm{kg})$ & $M=83,5, D P=9,8$ & $M=80,1, D P=13,8$ & 0,314 \\
\hline IMC $\left(\mathrm{kg} / \mathrm{m}^{2}\right)$ & $M=31,2, D P=2,7$ & $M=30,6, D P=2,3$ & 0,404 \\
\hline Circunferência da cintura $(\mathrm{cm})$ & $M=102,2, D P=6,4$ & $M=101,9, D P=8,3$ & 0,902 \\
\hline \multicolumn{4}{|l|}{ Pressão arterial $(\mathrm{mmHg})$} \\
\hline Sistólica & $M=134,0, D P=16,4$ & $M=142,0, D P=24,5$ & 0,156 \\
\hline Diastólica & $M=83,0, D P=8,8$ & $M=83,0, D P=14,1$ & 0,887 \\
\hline Consumo energético $(\mathrm{kcal})^{\mathbf{b}}$ & $M=2239,0, D P=600,0$ & $M=1872,0, D P=489,0$ & 0,955 \\
\hline
\end{tabular}

a Sete classes socioeconômicas em um gradiente decrescente de poder de compra de acordo com os critérios da Sociedade de Pesquisa de Mercado.

M: média; DP: desvio-padrão, ${ }^{\mathbf{b}} \mathrm{HAS}$ : hipertensão arterial; ${ }^{\mathrm{c}} \mathrm{DM}$ : diabetes Mellitus; ${ }^{\mathbf{d}}$ média de consumo determinada pelo registro alimentar de três dias.

Tabela 2. Barreiras referidas como limitantes para um ótimo nível de adesãoa nos grupos de Aconselhamento Nutricional em Grupo (ANG) e Atendimento Padrão Individual (API). Brasília (DF), 2005.

\begin{tabular}{|c|c|c|c|c|}
\hline \multirow[t]{2}{*}{ Variável } & \multicolumn{2}{|c|}{$\begin{array}{c}\text { Frequência de barreiras } \\
\text { referidas }(\%)\end{array}$} & \multirow{2}{*}{$\begin{array}{l}\text { Razão de } \\
\text { Chances }\end{array}$} & \multirow{2}{*}{$\begin{array}{l}\text { Intervalo de } 95 \% \\
\text { de Confiança }\end{array}$} \\
\hline & ANG $n=14$ & API $n=11$ & & \\
\hline Ser a dieta diferente da alimentação da família & 14,3 & 18,2 & 0,673 & $(0,028-10,379)$ \\
\hline Não preparar sua própria refeição & 28,6 & 18,2 & 1,774 & $(0,216-22,338)$ \\
\hline Fazer refeições fora de casa & 27,8 & 46,7 & 0,719 & $(0,121-3,336)$ \\
\hline Ser a dieta de alto custo alto & 14,3 & 14,3 & 1,115 & $(0,064-16,348)$ \\
\hline Ter dificuldade de aplicar os conceitos & 33,3 & 20,0 & 1,232 & $(0,254-7,302)$ \\
\hline
\end{tabular}

a ótimo nível de adesão: 100\% de frequência às três consultas do atendimento individual para o grupo de atendimento padrão (API) e pelo menos sete presenças entre as três consultas ambulatoriais e as seis reuniões de aconselhamento para o grupo. 
Quando se investigaram os fatores preditores de adesão - definida pela conclusão do protocolo, nenhuma variável apresentou associação

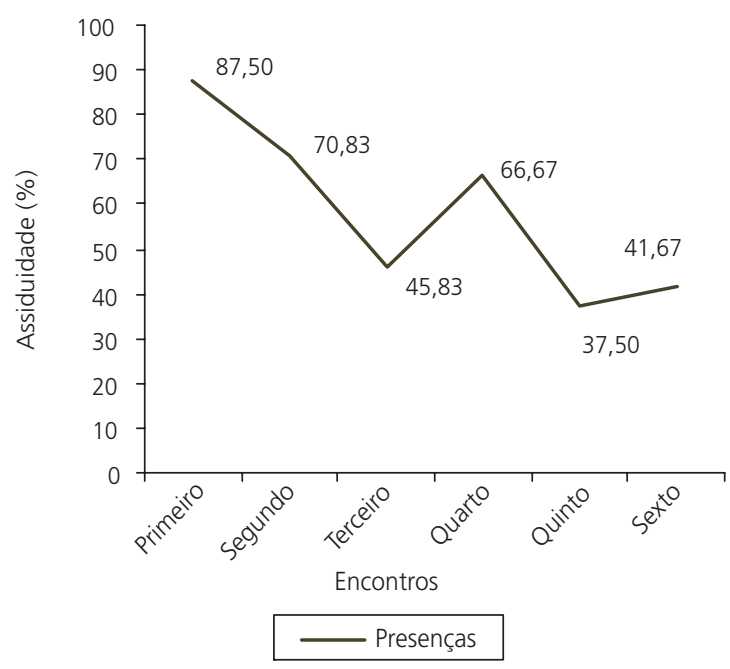

Figura 2. Assiduidade aos encontros entre os pacientes do grupo de aconselhamento nutricional $(n=24)$. Brasília (DF), 2005.

Nota: Primeiro encontro: Grupo alimentares e pirâmide dos alimentos; $2^{\circ}$ encontro: dez passos de uma alimentação saudável; $3^{\circ}$ encontro: rotulagem nutricional; $4^{\circ}$ encontro: composição de um prato saudável com participação da família; $5^{\circ}$ encontro: Dietas da moda; $6^{\circ}$ encontro: apresentação e discussão de um documentário sobre hábitos alimentares significativa que diferenciasse os voluntários que completaram dos que não completaram o estudo (resultados não apresentados).

A adesão também foi avaliada pela frequência às reuniões em grupo e às consultas. Foi considerada ótima quando houve $100 \%$ de frequência às três consultas do atendimento padrão individual e pelo menos sete presenças entre as três consultas ambulatoriais e as seis reuniões de aconselhamento para o grupo ANG. Com esse critério, apenas $45,8 \%$ e $40,7 \%$ dos participantes dos grupos ANG e API, respectivamente, atingiram esse nível de adesão. Os fatores mais citados pelos voluntários como barreira à adesão ao tratamento foram fazer refeições fora de casa (46,7\% - API) e dificuldade em aplicar os conhecimentos na prática, principalmente em eventos sociais (33,3\% - ANG) em ambos os grupos. Não foi evidenciada, contudo, nenhuma associação estatística entre as variáveis independentes após análise multivariada dos fatores limitantes para um ótimo nível de adesão dos dois grupos (Tabela 2).

De acordo com a análise do QFA simplificado, não houve diferença significativa entre os

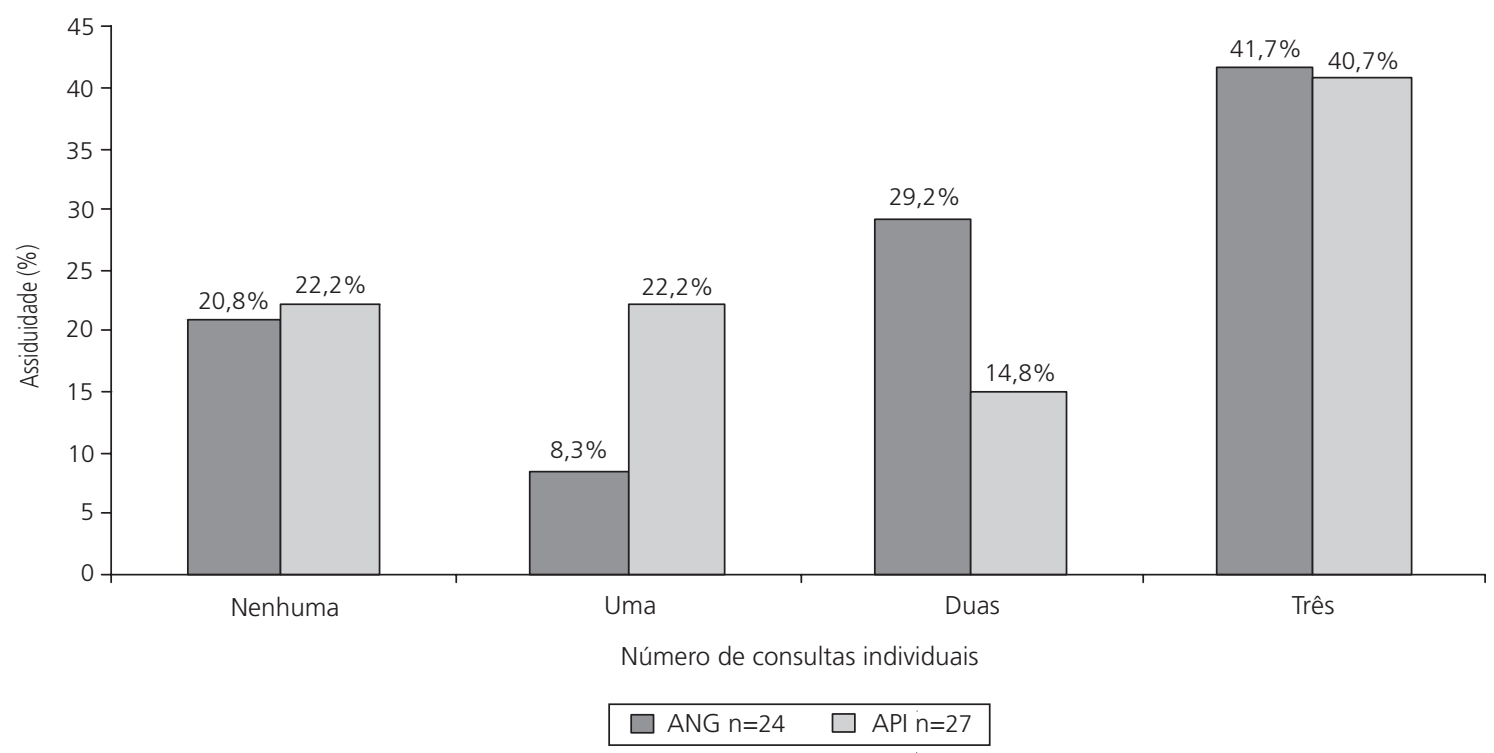

Figura 3. Assiduidade às consultas individuais ambulatoriais entre os pacientes dos grupos ANG e API. Brasília (DF), 2005. Nota: ANG: aconselhamento nutricional em grupo; API: atendimento padrão individual. 
$330 \mid$ N.G. GUIMARÃES et al.

grupos ao longo do tempo entre as variáveis estudadas. Verificou-se, no entanto, que o percentual médio do uso de temperos naturais e do número de refeições ao dia aumentou significativamente quando comparado à situação anterior e posterior à intervenção, com $p=0,0469$ e $p=0,0247$, respectivamente.

Com relação ao padrão alimentar quantitativo, houve redução média não significativa no consumo energético de $433 \mathrm{kcal}$ e de $416 \mathrm{kcal}$ em relação a situações anteriores e posteriores para o grupo ANG $(p=0,1018)$ e API $(p=0,0610)$, respectivamente. Quando realizada a análise entre os grupos, novamente a redução não foi estatisticamente significativa $(p=0,9550)$.

\section{I S C U S S Ã O}

O crescimento da incidência de Doenças Crônicas Não Transmissíveis (DCNT), observado nas últimas décadas, relaciona-se, em grande parte, com mudanças econômicas, sociais e demográficas que determinaram novos hábitos de vida, entre eles os comportamentos que desequilibram o balanço energético, induzindo ganho de peso, principalmente a alimentação inadequada e a redução da atividade física ${ }^{14,15}$.

Os grupos do presente estudo demonstravam um claro perfil de risco cardiovascular, compatível com os objetivos do trabalho. A randomização permitiu a comparabilidade entre os grupos. Notou-se que, tanto no grupo de aconseIhamento nutricional quanto no de atendimento padrão, houve predomínio de mulheres com alto nível de escolaridade e renda. Esse extrato da população parece ter acesso aos veículos de informação e demonstra preocupação com questões de saúde e estética e disposição para iniciar um tratamento clínico - convencional ou não, porém apresenta dificuldade em se manter em um programa de controle de peso.

Segundo dados mais recentes da Pesquisa de Orçamentos Familiares² , a evolução de excesso de peso e obesidade entre as mulheres dos grandes centros urbanos apresentou relativa estabi- lidade quando comparada às estimativas de pesquisa anterior realizada no País em 1989 (Pesquisa Nacional sobre Saúde e Nutrição - PNSN). O grau de instrução parece ter sido determinante para o controle da obesidade, apesar de os índices permanecerem altos para esse grupo populacional.

Bautista-Castaño et al. ${ }^{4}$ relataram que existe uma grande dificuldade e variabilidade na definição de critérios que retratem adequadamente a adesão ao tratamento da obesidade. Em geral, considera-se sucesso quando $20 \%$ a $45 \%$ dos participantes completam o protocolo. Nesse estudo, dois grupos de pacientes com excesso de peso foram acompanhados durante três meses, recebendo orientação nutricional específica para mudança no comportamento alimentar e, consequentemente, para redução dos fatores de risco cardiovascular. A adesão medida por meio da assiduidade ao tratamento foi de aproximadamente $40 \%$ tanto para o grupo submetido a um modelo API quanto para o grupo ANG.

Os indivíduos do grupo ANG participaram de seis reuniões em grupo, com discussão sobre alimentação saudável e atividade física. Notadamente, no quarto encontro, houve um aumento na assiduidade para 66,7\%; nesse dia, os familiares foram convidados a participar da reunião. Esses dados sugerem que a adesão pode ser facilitada quando se envolve a família em função do seu contexto e do suporte tão importantes para o manejo da obesidade ${ }^{16}$.

Contrariamente ao presente estudo, Rickheim et al. ${ }^{16}$ verificaram uma adesão de $72,0 \%$ após três meses de acompanhamento com pacientes diabéticos tipo 2 . Todos os indivíduos que completaram a pesquisa, independentemente da orientação recebida (aconselhamento individual ou em grupo), apresentaram melhora do comportamento alimentar. Da mesma forma, em estudo de intervenção com aconselhamento nutricional realizado por Aldana et al. ${ }^{17}$, a mudança no estilo de vida resultou em melhora significativa a curto prazo (um mês) nas variáveis dietéticas e metabólicas. Com uma amostra de 337 indivíduos, a taxa de desistência foi baixa entre os grupos estu- 
dados (7,2\% intervenção e 8,6\% controle), provavelmente devido à complexidade organizacional da intervenção, além de uma elevada motivação intrínseca e de um estilo de vida favorável às mudanças pelos participantes.

É comum encontrarmos referências de baixa adesão ao tratamento nutricional a curto e longo prazo. Inelmen et al. ${ }^{5}$, em protocolo com 383 pacientes em tratamento ambulatorial individual para obesidade, relataram que após o período de um ano, 296 pacientes haviam desistido do estudo: $69,7 \%$ desses abandonaram o tratamento ao final dos primeiros três meses e somente $22,7 \%$ dos pacientes completaram todo o protocolo proposto. Segundo os autores, o insucesso do tratamento foi associado ao tipo de estratégia utilizada: o atendimento individual não foi suficiente para motivar os indivíduos; eles sugerem que um programa com reuniões em grupo periódicas seria uma medida eficaz para melhorar o nível de adesão. Diferentes autores apontam outras estratégias para diminuir as perdas inerentes ao tratamento convencional: estabelecer metas realistas, programas que associem atividade física e educação nutricional, além do suporte social e familiar ${ }^{4,17,18}$, ou sugerem ainda o estabelecimento de uma aliança terapêutica que dê ao paciente a possibilidade de solucionar problemas relacionados à alimentação ${ }^{6}$.

Em estudo prospectivo realizado por Teixeira et al. ${ }^{19}$, foram acompanhadas 158 mulheres adultas com excesso de peso submetidas à terapia em grupo para mudança de comportamento, e verificaram-se como principais motivos de desistência nos primeiros quatro meses do tratamento: falta de tempo para a participação no programa, insatisfação com o programa, problemas pessoais e limitações de saúde. No estudo em Brasília, as perdas iniciais e no seguimento tiveram características semelhantes: problemas familiares, pessoais e de saúde, desinteresse e não comparecimento às entrevistas e consultas por falta de tempo.

Ainda não estão bem esclarecidos os motivos pelos quais alguns indivíduos adotam e sustentam comportamentos que contribuem para a perda de peso e sua manutenção e outros não. Estudos sugerem como fatores preditores de boa adesão: ter elevado grau de motivação intrínseca, manter assiduidade aos encontro ${ }^{20}$, ser mais ativo $^{19}$, ser do sexo masculino, ter idade avançada, apresentar IMC menor, ausência de tratamentos anteriores e de história familiar de obesidade 4 . Dentre as variáveis analisadas neste estudo, nenhuma foi considerada como fator preditor para adesão; possivelmente esses resultados são consequência das perdas amostrais, resultando em diminuição do poder estatístico da amostra final.

Com relação aos fatores referidos como barreira para adesão ótima às orientações recebidas ao longo do estudo, eles foram bastante variados em ambos os grupos; mais uma vez, possivelmente, o tamanho amostral não tenha sido suficiente para detectar os determinantes de baixa adesão. Fazer refeições fora de casa e dificuldade em aplicar, na prática, os conhecimentos, principalmente em eventos sociais, foram os fatores limitantes mais citados pelos voluntários deste estudo. Para Rickheim et al. ${ }^{16}$ e Schlundt et al. ${ }^{21}$, a adesão relaciona-se com fatores inseridos no contexto domiciliar, de trabalho e social. As desistências podem estar relacionadas também ao maior número de doenças associadas e à depressão ${ }^{5}$. Neste estudo, esses fatores não foram analisados.

O nível elevado de conhecimento, antes da intervenção, parece ser compatível com o elevado grau de escolaridade e renda da população estudada e não sofreu alteração após o acompanhamento. Esse perfil pode explicar os resultados diferentes obtidos em outras pesquisas, que evidenciaram melhora do nível de conhecimento com aplicação de modelos de intervenção ${ }^{16,17}$.

Qualitativamente, as orientações recebidas tanto individualmente quanto pelas dinâmicas de grupo repercutiram no padrão alimentar pelo menos com relação ao cumprimento de todas as refeições, inclusive o café da manhã e a diminuição do consumo de sal, dando preferência por 
outros temperos naturais. Parece que pequenas modificações do padrão alimentar, mesmo não alterando a curto prazo o estado de saúde, podem ser alcançadas com modelos de aconselhamento nutricional ${ }^{16,22,23}$. As repercussões clínicas e metabólicas dependem de um período maior de intervenção, o que não estava previsto no presente estudo.

Com relação ao impacto do tratamento da obesidade no consumo energético, no estudo de Kearney \& McElhone ${ }^{24}$ não foram encontradas reduções significativas da ingestão quantitativa após três meses. Os autores referem que os indivíduos obesos apresentam inabilidade em avaliar adequadamente o padrão alimentar, podendo subestimar o valor energético da dieta habitual principalmente por considerarem seu padrão de ingestão suficientemente saudável. Johnson et al. ${ }^{25}$, após seis meses de acompanhamento de mulheres com sobrepeso e obesidade, verificaram que o sub-relato é maior após o término de programas para perda de peso, sugerindo que essa alteração aconteça para coincidir com as recomendações recebidas no decorrer do tratamento. O consumo energético inicial dos participantes do presente estudo também parece ter sido subestimado, sendo incompatível com o grau de obesidade apresentado. Por sua vez, a redução do consumo observada após três meses de acompanhamento, em aproximadamente 400kcal, não foi estatisticamente significativo e, apesar de estar de acordo com as orientações recebidas, mais uma vez pode não refletir a realidade.

Os resultados mostraram que a despeito do emprego de um modelo de intervenção com abordagem diferenciada, aplicação de dinâmicas de grupo e conteúdo diversificado, o aconselhamento nutricional em grupo proposto obteve adesão compatível com resultados da literatura, porém insuficiente para determinar nível maior ou diferenciado quando comparado ao grupo controle de atendimento padrão. Verificou-se que independentemente do tipo de acompanhamento, houve pouca repercussão nas práticas alimentares. Alguns programas podem falhar diante da com- plexidade do problema e das inúmeras dificuldades impostas no ambiente urbano atual. O tempo ideal de intervenção, o modelo a ser empregado para públicos distintos, assim como envolvimento de suporte social e familiar ainda não estão completamente esclarecidos e podem ter influenciado os resultados desse estudo. Como a obesidade é uma desordem heterogênea e multifatorial, a identificação de características individuais com aprofundamento da abordagem comportamental continuada e multiprofissional deve ser objeto de mais investigação.

\section{COLABORADORES}

N.G. GUIMARÃES participou da concepção, do desenho, da coleta de dados, da análise, da interpretação dos dados e da redação do artigo. E.S. DUTRA e M.K. ITO participaram do desenho da pesquisa e da análise e da interpretação dos dados. K.M.B. CARVALHO, orientadora do trabalho, participou das etapas de planejamento da pesquisa, da análise e interpretação dos dados e da redação do artigo.

\section{REFERÊ NCIAS}

1. World Health Organization. Diet, nutrition and prevention of chronic diseases. Report of a Joint WHO/FAO Expert Consultation. Geneva: WHO; 2003. WHO Technical Report Series, 916.

2. Instituto Brasileiro de Geografia e Estatística. Pesquisa de orçamentos familiares 2002-2003: análise da disponibilidade domiciliar de alimentos e do estado nutricional no Brasil. Rio de Janeiro: IBGE; 2004.

3. Goldstein DJ. Beneficial health effects of modest weight loss. Int J Obes Relat Metab Disord. 1992; 16(6):397-415.

4. Bautista-Castaño I, Molina-Cabrillana J, MontoyaAlonso JA, Serra-Majem L. Variables predictive of adherence to diet and physical activity recommendations in the treatment of obesity and overweight, in a group of spanish subjects. Int J Obes Relat Metab Disord. 2004; 28(5):697-705.

5. Inelmen EM, Toffanello ED, Enzi G, Gasparini G, Mioto F, Sergi G, et al. Predictors of drop-out in overweight and obese outpatients. Int J Obes (London). 2005; 29(1):122-8. 
6. Rodrigues EM, Soares FPTP, Boog MCF. Resgate do conflito de aconselhamento no contexto do atendimento nutricional. Rev Nutr. 2005; 18(1): 119-28. doi: 10.1590/s1415-5273200500010 0011.

7. Brasil. Ministério da Saúde. Antropometria: como pesar e medir. [acesso 2004 nov]. Disponível em: <htpp://dtr2004.saude.gov.br/nutricao/ documentos/album_antropometria.pdf>.

8. Sociedade Brasileira de Cardiologia. III Consenso Brasileiro de Hipertensão Arterial. Hiperativo. 1999; 6(1):67-106.

9. Sociedade Brasileira de Pesquisa de Mercado. Critério de classificação econômica Brasil. [acesso 2004 jan 10]. Disponível em: <htpp://www.sbpm. org.br>.

10. Philippi ST. Tabela de composição de alimentos: suporte para decisão nutricional. São Paulo; 2001.

11. Pinheiro ABV, Lacerda EMA, Benzecry EH, Gomes MCS, Costa VM. Tabela para avaliação de consumo alimentar em medidas caseiras. São Paulo: Atheneu; 2000.

12. Holli BB, Calabrese RJ, Maillet JO. Communication and education skills for dietetics professionals $4^{\text {th }}$ ed. Philadelphia: Lippincott Williams and Wilkins; 2003.

13. Statistics Analisys System. [software]. Statistics Analisys Institute. Cary (NC): SAS Institute Inc; 2004.

14. Drewnowski A, Popkin BM. The nutrition transition: new trends in the global diet. Nutr Rev. 1997; 55(2): 31-43.

15. World Health Organization. Global strategy on diet, physical activity and health. Resolution WHA57.17. The 57th World Health Assembly. Geneva: WHO; 2004.

16. Rickheim PL, Weaver TW, Flader JI, Kendall DM. Assessment of group versus individual diabetes education: a randomized study. Diabetes Care. 2002; 25(2):269-74.

17. Aldana SG, Greenlaw RL, Diehl HA, Salberg A, Merrill RM, Ohimine $S$, et al. Effects of an intensive diet and physical activity modification program on the health risks of adults. J Am Diet Assoc. 2005; 105(3):371-81.

18. Andersson I, Rössner S. Weight development, drop-out pattern and changes in obesity-related risk factors after two years treatment of obese men. Int J Obes Relat Metab Disord. 1997; 21(3):211-6.

19. Teixeira PJ, Going SB, Houtkooper LB, Cussler EC, Metcalfe LL, Blew R M, et al. Pretreatment predictors of attrition and successful weight management in women. Int J Obes Relat Metab Disord. 2004; 28(9):1124-33.

20. Wadden TA, Foster GD, Wang J, Pierson RN, Yang $\mathrm{MU}$, Moreland K, et al. Clinical correlates of shortand long-term weight loss. Am J Clin Nutr. 1992; 56(1Suppl):271S-4S.

21. Schlundt DG, Rea MR, Kline SS, Pichert JW. Situational obstacles to dietary adherence for adults with diabetes. J Am Diet Assoc. 1994; 94(8): 874-6, 879; quiz 877-8.

22. Appel LJ, Moore TJ, Obarzanek E, Vollmer WM, Svetkey LP, Sacks FM, et al. The effect of dietary patterns on blood pressure: results from the Dietary Approaches to Stop Hypertension (DASH) clinical trial. N Engl J Med. 1997; 336(16):1117-24.

23. Appel LJ, Champagne CM, Harsha DW, Cooper LS, Obarzadek E, Elmer PJ, et al. Writing Group of the PREMIER Collaborative Research Group. Effects of comprehensive lifestyle modification on blood pressure control: main results of the PREMIER clinical trial. JAMA. 2003; 289(16):2083-93.

24. Kearney JM, McElhone S. Perceived barriers in trying to eat healthier: results of a pan-EU consumer attitudinal survey. Br J Nutr. 1999; 81(2Suppl): 133S-7S.

25. Johnsom RK, Friedman AB, Harvey-Berino J, Gold $B C$, McKenzie D. Participation in a behavioral weight-loss program worsens the prevalence and severity of underreporting among obese and overweight women. J Am Diet Assoc. 2005; 105(12):1948-51.

Recebido em: 28/7/2008

Versão final reapresentada em: 13/7/2009 Aprovado em:18/3/2010 